\title{
POSITIVELY CURVED TOTALLY REAL MINIMAL SUBMANIFOLDS IMMERSED IN A COMPLEX PROJECTIVE SPACE
}

\author{
KOICHI OGIUE ${ }^{1}$
}

\begin{abstract}
ABSTRACr. A sufficient condition for a complete totally real minimal submanifold immersed in a complex projective space to be totally geodesic is given in terms of sectional curvature.
\end{abstract}

1. Statement of result. Let $P_{n}(C)$ be an $n$-dimensional complex projective space with the Fubini-Study metric of constant holomorphic sectional curvature $c$, and let $M$ be an $n$-dimensional complete totally real minimal submanifold immersed in $P_{n}(C)$.

The purpose of this paper is to prove

THEOREM. If the sectional curvature of $M$ is greater than $(n-2) c / 4(2 n-1)$, then $M$ is totally geodesic.

The result for $n=2$ was proved by S. T. Yau [3].

2. Basic lemmas. We use the same notation and terminologies as in [1] unless otherwise stated. It was proved in [1] that the second fundamental form of the immersion satisfies

$$
\begin{aligned}
\frac{1}{2} \Delta\|\sigma\|^{2}= & \left\|\nabla^{\prime} \sigma\right\|^{2}+\sum_{i, j, k, l, m}\left(h_{i j}^{m^{*}} h_{k l}^{m^{*}} R_{l i j k}+h_{i j}^{m^{*}} h_{i l}^{m^{*}} R_{l k j k}\right) \\
& +\frac{1}{2} \sum_{i, j} \operatorname{tr}\left(A_{i^{*}} A_{j^{*}}-A_{j^{*}} A_{i^{*}}\right)^{2}+\frac{c}{4}\|\boldsymbol{\sigma}\|^{2} .
\end{aligned}
$$

On the one hand, using the equation of Gauss we obtain

$$
\begin{aligned}
\sum_{i, j, k, l, m}\left(h_{i j}^{m^{*}}\right. & \left.h_{k l}^{m^{*}} R_{l i j k}+h_{i j}^{m^{*}} h_{i l}^{m^{*}} R_{l k j k}\right) \\
& =\frac{n c}{4}\|\sigma\|^{2}+\frac{1}{2} \sum_{i, j} \operatorname{tr}\left(A_{i^{*}} A_{i^{*}}-A_{j^{*}} A_{i^{*}}\right)^{2}-\sum_{i, j}\left(\operatorname{tr} A_{i^{*}} A_{j^{*}}\right)^{2} .
\end{aligned}
$$

On the other hand, Yau's idea in [4] can be applied as follows: For each $m$, let $h_{1}^{m}, \ldots, h_{n}^{m}$ be the eigenvalues of $A_{m^{*}}$. Then we have

Received by the editors March 11, 1975.

AMS (MOS) subject classifications (1970). Primary 53C40.

Key words and phrases. Totally real submanifold, minimal submanifold, complex projective space, totally geodesic.

${ }^{1}$ Partially supported by NSF Grant GP-36684 and the Matsunaga Science Foundation. 


$$
\begin{aligned}
\sum_{i, j, k, l}\left(h_{i j}^{m^{*}} h_{k l}^{m^{*}} R_{l i j k}+h_{i j}^{m^{*}} h_{i l}^{m^{*}} R_{l k j k}\right) & =\sum_{i, k}\left\{h_{i}^{m} h_{k}^{m} R_{k i i k}+\left(h_{i}^{m}\right)^{2} R_{i k i k}\right\} \\
& =\frac{1}{2} \sum_{i, k}\left(h_{i}^{m}-h_{k}^{m}\right)^{2} R_{i k i k} .
\end{aligned}
$$

Therefore if the sectional curvature of $M$ is greater than $\delta$, we have

$$
\begin{aligned}
\sum_{i, j, k, l}\left(h_{i j}^{m^{*}} h_{k l}^{m^{*}} R_{l j k}+h_{i j}^{m^{*}} h_{i l}^{m^{*}} R_{l k j k}\right) & \geqslant \frac{1}{2} \sum_{i, k}\left(h_{i}^{m}-h_{k}^{m}\right)^{2} \delta \\
& =n \delta \sum_{i}\left(h_{i}^{m}\right)^{2}=n \delta \operatorname{tr} A_{m^{*}}^{2},
\end{aligned}
$$

from which it follows that

$$
\sum_{i, j, k, l, m}\left(h_{i j}^{m^{*}} h_{k l}^{m^{*}} R_{l i j k}+h_{i j}^{m^{*}} h_{i l}^{m^{*}} R_{l k j k}\right) \geqslant n \delta\|\sigma\|^{2} .
$$

From (1), (2) and (3) we have

LEMMA 1. If the sectional curvature of $M$ is greater than $\delta$, then

$$
\begin{aligned}
\frac{1}{2} \Delta\|\sigma\|^{2} \geqslant & \left\|\nabla^{\prime} \sigma\right\|^{2}+(1+a) n \delta\|\sigma\|^{2}-\frac{n a-1}{4} c\|\sigma\|^{2} \\
& +\frac{1-a}{2} \sum_{i, j} \operatorname{tr}\left(A_{i^{*}} A_{j^{*}}-A_{j^{*}} A_{i^{*}}\right)^{2}+a \sum_{i, j}\left(\operatorname{tr} A_{i^{*}} A_{j^{*}}\right)^{2}
\end{aligned}
$$

for $a \geqslant-1$.

The following lemma is purely algebraic.

Lemma 2. $n^{-1}\|\sigma\|^{4} \leqslant \sum_{i, j}\left(\operatorname{tr} A_{i^{*}} A_{j^{*}}\right)^{2} \leqslant\|\sigma\|^{4}$.

3. Proof of theorem. Since the symmetric $(n, n)$-matrix $\left(\operatorname{tr} A_{i^{*}} A_{j^{*}}\right)$ is covariant for the change of basis, for a suitable choice of basis we may assume that

$$
\operatorname{tr} A_{i^{*}} A_{j^{*}}=0 \quad \text { for } i \neq j .
$$

An algebraic lemma of Chern, doCarmo and Kobayashi (Lemma 1 in [2]) implies that

$$
\begin{aligned}
\sum_{i, j} \operatorname{tr}\left(A_{i^{*}} A_{j^{*}}-A_{j^{*}} A_{i^{*}}\right)^{2} & \geqslant-2 \sum_{i \neq j} \operatorname{tr} A_{i^{*}}^{2} \operatorname{tr} A_{j^{*}}^{2} \\
& =-2\|\boldsymbol{\sigma}\|^{4}+2 \sum_{i}\left(\operatorname{tr} A_{i^{*}}^{2}\right)^{2} .
\end{aligned}
$$

From Lemma 1, (4) and (5), it follows that

$$
\frac{1}{2} \Delta\|\sigma\|^{2} \geqslant(1+a) n \delta\|\sigma\|^{2}-\frac{n a-1}{4} c\|\sigma\|^{2}-(1-a)\|\sigma\|^{4}+\sum_{i}\left(\operatorname{tr} A_{i^{*}}^{2}\right)^{2}
$$

for $-1 \leqslant a \leqslant 1$.

This, together with Lemma 2 and (4), implies that 


$$
\frac{1}{2} \Delta\|\sigma\|^{2} \geqslant\left\{(1+a) n \delta-\frac{n a-1}{4} c\right\}\|\sigma\|^{2}+\left\{\frac{1}{n}-(1-a)\right\}\|\sigma\|^{4} .
$$

In particular, putting $a=1-1 / n$, we obtain

$$
\frac{1}{2} \Delta\|\sigma\|^{2} \geqslant\left\{(2 n-1) \delta-\frac{n-2}{4} c\right\}\|\sigma\|^{2},
$$

from which the theorem follows.

\section{REFERENCES}

1. B. Y. Chen and K. Ogiue, On totally real submanifolds, Trans. Amer. Math. Soc. 193(1974), 257-266.

2. S. S. Chern, M. P. do Carmo and S. Kobayashi, Minimal submanifolds of a sphere with second fundamental form of constant length, Functional Analysis and Related Fields (Proc. Conf. for M. Stone, Univ. of Chicago, Chicago, Ill., 1968), Springer, New York, 1970, pp. 59-75. MR 42 \# 8424.

3. S. T. Yau, Submanifolds with constant mean curvature. I, Amer. J. Math. 96(1974), 346-366.

4. _- Submanifolds with constant mean curvature. II, Amer. J. Math. 97 (1975), 76-100.

Department of Mathematics, Tokyo Metropolitan University, Tokyo, Japan 